\title{
Recurrent Endophthalmitis after Trabeculectomy for Glaucoma: Importance of Timely Filtering Bleb Coverage for the Conjunctiva
}

\author{
Carl-Ludwig Schönfeld \\ Augenklinik Herzog Carl Theodor, Munich, Germany
}

\section{Key Words}

Glaucoma surgery · Complications · Endophthalmitis · Vitrectomy · Filtering bleb

\begin{abstract}
Purpose: To report the occurrence of endophthalmitis after trabeculectomy for glaucoma that was treated by vitrectomy without bleb closure and recurred 3 times after an initial clinically inapparent period of about 2 years.
\end{abstract}

Methods: Interventional case report and short review of the pertinent literature.

Results: A 73-year-old Caucasian female underwent trabeculectomy (without mitomycin) for glaucoma. Four years after the trabeculectomy, an endophthalmitis with Staphylococcus epidermidis as the causative organism occurred and was treated with a pars plana vitrectomy (20-gauge) and a combined antibiotic and anti-inflammatory drug regimen. The bleb was not covered during the emergency procedure because the filtering bleb was heavily infected and filled with pus. As the filtering bleb healed nicely and the filtering function was restored, the filtering bleb was left as it was, and best-corrected visual acuity initially recovered to 0.8 . After 22 months, the endophthalmitis recurred, this time with Enterococcus faecalis as the causative organism. Its treatment required a total of four further vitrectomies (23-gauge), each accompanied by the same antibiotic and antiinflammatory drug regimen that had been applied previously. However, the visual acuity could not be preserved, and the eye is blind with defective light projection.

Conclusions: The literature suggests an aggressive surgical approach to endophthalmitis, and the present case report confirms this. Since the visual prognosis of eyes after endophthalmitis is poor, the course of treatment for endophthalmitis after trabeculectomy should emphasize recurrence prophylaxis rather than address glaucoma symptoms and therefore include safe bleb leak coverage. 


\section{Introduction}

Glaucoma surgery, albeit showing a generally encouraging efficacy and safety profile, is faced with a classic dilemma: the more effective a surgical method, the more likely are bleb-related infections. The filtering bleb as such hampers the protective function of the conjunctiva against microbial invasion due to its stretching and thinning, and the trabeculectomy provides a potential migration path for microorganisms from the bleb via the anterior chamber into the vitreous; moreover, adjuvant antifibrotic agents as well as sub-optimal surgical proceedings in terms of bleb placement and patching increase the likelihood for a clinical manifest infection, i.e. blebitis and/or endophthalmitis $[1,2]$.

Endophthalmitis is the most severe and most dreaded infectious complication in ocular surgery and can occur with a substantial delay of several years after surgery. The spectrum of causative organisms is broad and includes typical skin bacteria as well as nosocomial pathogens that may unveil hospital hygiene deficiencies [2-6]. While the treatment of endophthalmitis with regular- and small-gauge vitrectomy is possible [7, 8], there are no established standard operating procedures $[9,10]$, and the visual outcome is generally poor even if the eye itself can be preserved $[2-4,11,12]$. The latter statement is especially true with regard to recurrent and/or chronic endophthalmitis [13-15], and therefore recurrence prophylaxis should have a very high priority in treatment decision-making.

\section{Case Report}

A 73-year-old Caucasian female underwent trabeculectomy (without mitomycin) for glaucoma that had occurred approximately 15 years after buckling surgery for retinal detachment. 18 months after the trabeculectomy, phacoemulsification was performed.

Almost exactly 4 years after the trabeculectomy, an endophthalmitis with Staphylococcus epidermidis as the causative organism occurred. The endophthalmitis was treated with a pars plana vitrectomy (20-gauge) and a combined antibiotic and anti-inflammatory drug regimen, using intravitreal vancomycin $1.0 \mathrm{mg}$, amikacin $0.4 \mathrm{mg}$ and dexamethasone $1.0 \mathrm{mg}$. The bleb was not covered during the emergency procedure because the filtering bleb was heavily infected and filled with pus. Postoperative local treatment comprised moxifloxacin, chloramphenicol and prednisolonacetat drops every $30 \mathrm{~min}$ and latanoprost at night. Systemic treatment comprised imipenem $500 \mathrm{mg} 3$ times per day, ciprofloxacin $500 \mathrm{mg} 3$ times per day and prednisolone acetate $100 \mathrm{mg} /$ day for 3 days, the latter subsequently phased out by a dose reduction of $20 \mathrm{mg}$ every 3 days. Since the filtering bleb healed nicely and the filtering function was restored, it was decided to leave the filtering bleb as it was. The Seidel test was negative.

After an initial remission of approximately 22 months, the endophthalmitis recurred, this time with Enterococcus faecalis as the causative organism. Its treatment required a total of four further vitrectomies (23-gauge), each accompanied by the same antibiotic and anti-inflammatory drug regimen that had been applied previously. After the initial vitrectomy, the visual acuity had recovered to 0.8 . Three days before the second vitrectomy for recurrent endophthalmitis, the visual acuity was still 0.6 , but it deteriorated to light perception immediately before the procedure. Before and after subsequent vitrectomies for recurrent endophthalmitis, the patient was at best able to count fingers, and ultimately the eye remained blind with defective light projection. 


\section{Discussion}

Antifibrotic agents such as mitomycin are causally involved in the majority of cases of post-trabeculectomy endophthalmitis [2], but the present case underlines the fact that it can occur without antifibrotic adjuvants.

The sporadic occurrence of endophthalmitis after ocular surgery procedures is no sign of erroneous proceeding as such, although it may indicate technical and/or hygienic faults. However, the literature suggests that an aggressive surgical approach to endophthalmitis is warranted $[1,13]$, and the present case report confirms this. Recurrent endophthalmitis is an unmistakable sign of persistence of microorganisms in the eye or, more likely, a re-infection after primarily successful treatment. Therefore, the initial treatment approach should place special emphasis on the avoidance of loci minoris resistentiae for germ invasion.

This is obviously contradictory to the treatment goals of glaucoma where the construction of the filtering bleb facilitates germ invasion. However, since the visual prognosis of eyes after endophthalmitis is poor [2-4,11,12] - and even much more so after recurrence [13-15] -, the course of treatment for endophthalmitis after trabeculectomy should emphasize recurrence prophylaxis and therefore include a safe bleb leak coverage [16].

\section{Acknowledgment}

This case report was supported by a grant from the Herzog Carl Theodor Eye Clinic Foundation, Munich, Germany.

\section{Disclosure Statement}

The author has no conflicts of interest to declare. 


\section{References}

1 Mac I, Soltau JB: Glaucoma-filtering bleb infections. Curr Opin Ophthalmol 2003;14:91-94.

2 Song A, Scott IU, Flynn HW Jr, Budenz DL: Delayed-onset bleb-associated endophthalmitis: clinical features and visual acuity outcomes. Ophthalmology 2002;109:985-991.

3 Al-Turki TA, Al-Shahwan S, Al-Mezaine HS, Kangave D, Abu El-Asrar AM: Microbiology and visual outcome of bleb-associated endophthalmitis. Ocul Immunol Inflamm 2010;18:121-126.

4 Leng T, Miller D, Flynn HW Jr, Jacobs DJ, Gedde SJ: Delayed-onset bleb-associated endophthalmitis (1996-2008): causative organisms and visual acuity outcomes. Retina 2011;31:344-352.

5 Pierre DJ, Tang J: Bleb associated endophthalmitis with methicillin-resistant Staphylococcus aureus. Br J Ophthalmol 2010;94:390-392.

6 Ye H, Sun X, Gan D, Yu X, Zhou W, Xu G, Jiang R: Bleb-associated endophthalmitis in a Chinese population (2003-2010): clinical characteristics and visual outcome. Eur J Ophthalmol 2012;22:719-725.

7 Almanjoumi AM, Combey A, Romanet JP, Chiquet C: 23-gauge transconjunctival sutureless vitrectomy in treatment of post-operative endophthalmitis. Graefes Arch Clin Exp Ophthalmol 2012;250:1367-1371.

8 Endophthalmitis Vitrectomy Study Group: Results of the Endophthalmitis Vitrectomy Study. A randomized trial of immediate vitrectomy and of intravenous antibiotics for the treatment of postoperative bacterial endophthalmitis. Arch Ophthalmol 1995;113:1479-1496.

9 Behrens-Baumann W: Zur Prophylaxe und Therapie der postoperativen Endophthalmitis. ESCRS-Studie und 'Early Vitrectomy Study' in der Kritik. Ophthalmologe 2011;108:1062, 1064-1066.

10 Chiam PJ, Arashvand K, Shaikh A, James B: Management of blebitis in the United Kingdom: a survey. Br J Ophthalmol 2012;96:38-41.

11 Ding Y, Lin M, Liu H, Zhang W, Wang L, Li Y: Outcomes of post-cataract surgery endophthalmitis referred to a tertiary center from local hospitals in the south of China. Infection 2011;39:451-460.

12 Rahimi M, Ghassemifar V, Nowroozzadeh MH: Outcome of endophthalmitis treatment in a tertiary referral center in southern Iran. Middle East Afr J Ophthalmol 2012;19:107-114.

13 Maalouf F, Abdulaal M, Hamam RN: Chronic postoperative endophthalmitis: a review of clinical characteristics, microbiology, treatment strategies, and outcomes. Int J Inflam 2012;2012:313248.

14 Miller KV, Eisley KM, Shanks RM, Lahr RM, Lathrop KL, Kowalski RP, Noecker RJ: Recurrent enterococcal endophthalmitis seeded by an intraocular lens biofilm. J Cataract Refract Surg 2011;37:1355-1359.

15 Teoh SC, Lee JJ, Chee CK, Au Eong KG: Recurrent Enterococcus faecalis endophthalmitis after phacoemulsification. J Cataract Refract Surg 2005;31:622-626.

16 Kosmin AS, Wishart PK: A full-thickness scleral graft for the surgical management of a late filtration bleb leak. Ophthalmic Surg Lasers 1997;28:461-468. 